\title{
Reflets
}

Revue ontaroise d'intervention sociale et communautaire

\section{Une Alliance avec la vie}

\section{Carole Labrèche}

Volume 1, numéro 2, automne 1995

La santé communautaire en Ontario français : défis et espoirs

URI : https://id.erudit.org/iderudit/026082ar

DOI : https://doi.org/10.7202/026082ar

Aller au sommaire du numéro

Éditeur(s)

Reflets : Revue ontaroise d'intervention sociale et communautaire

ISSN

1203-4576 (imprimé)

1712-8498 (numérique)

Découvrir la revue

Citer cet article

Labrèche, C. (1995). Une Alliance avec la vie. Reflets, 1(2), 166-170.

https://doi.org/10.7202/026082ar

Tous droits réservés (C) Reflets : Revue ontaroise d'intervention sociale et communautaire, 1995

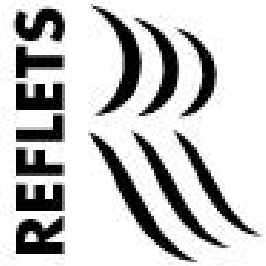

Ce document est protégé par la loi sur le droit d'auteur. L'utilisation des services d'Érudit (y compris la reproduction) est assujettie à sa politique d'utilisation que vous pouvez consulter en ligne.

https://apropos.erudit.org/fr/usagers/politique-dutilisation/ 


\section{Une Alliance avec la vie}

\section{C arole L abrèche}

A lliance des francophones en santé mentale, 0 ttawa

$\mathrm{N}$ ous vivons dans une société complexe où les systèmes de valeurs coexistent et souvent s'affrontent. $D$ ans ce rapide déferlement desévénements et deschoses, il faut lutter fort pour survivre. Ceux qui ne réussissent pas risquent de connaitre des démêlés avec la psychiatrie. $\mathrm{N}$ ous sommes nombreux à craquer à chaque année sous le poids de problèmes socio-économiques. II nous arrive de nous rétablir, mais ce n'est pas toujours le cas. N ous tentons alors d'apaiser nos tourments et de nous oublier.

L'Alliance des francophones en santé mentale d'O ttawa$C$ arleton ne veut pas oublier ces hommes et ces femmes qui ont subi les soins du milieu hospitalier. Le 16 juin 1994 avait lieu, au Patro d'O ttawa, le premier colloque annuel des francophones en santé mentale. C'est sous le thème Prendre la parole que s'est déroulée cette journée de sensibilisation. Le mot d'ordre était lancé: briser le silence dont sont victimes les personnes ayant un vécu psychiatrique. Puisque le concept de santé mentale demeure un immense fourre-tout, il est parfois difficile de s'y retrouver. Q ui consulter quand ça va mal? Psychiatre? Psychologue?T hérapeute? $\mathrm{Q}$ ue penser de la médication, de la surmédication? Q uels sont les effets à long terme des pilules pour les nerfs? Q uels sont les droits des personnes en matière de traitement? Comment faire face à l'angoisse, à la peur, à la solitude et au stress?

Autant de questions auxquelles seront confrontés bien des gens durant leur vie. N ous voyons alors combien il est important de renseigner les personnes afin qu'elles puissent transformer leurs problèmes de santé mentale en stratégies de vie. A insi, la personne qui traverse des difficultés peut se donner les outils qui lui 
«P rogrammés comme nous le sommes pour la réussite, quand les problèmes de santé mentale surviennent, nous n'avons qu' une envie: nous isoler, nous refermer comme des huîtres.» manquent pour fonctionner. Elle sait qu'il existe des ressources dans la communauté et peut alors éviter le piège de l'isolement.

Programmés comme nous le sommes pour la réussite, quand les problèmes de santé mentale surviennent, nousn'avons qu'une envie: nous isoler, nous refermer comme des huitres. N ous sommes coincés dans la souffrance. $\mathrm{N}$ ous nous sentons au plus bas de l'échelle, diminués, moins que rien. C ombien de personnes sont retournées chez elles après une hospitalisation, débarrassées de certains de leurs symptômes, mais complètement désarmées devant la vie? U ne transition s'impose pour tous ces poqués du système médical. II leur faut un lieu pour retrouver leur forme affective et leur autonomie. C'est là que l'Alliance entre en jeu.

Bien faire quelque chose, utiliser ses talents, se sentir utile sont des besoins bien légitimes. À I'Alliance, nous nous donnons un projet et nous travaillons ensemble à le réaliser. La B ouffe onrit fait partie de ces réalisations. $N$ os habitudes alimentaires étant ce qu'elles sont - nousnégligeons souvent l'importance de bien manger surtout lorsque nous sommes malades - I'Alliance a déjà organisé des soupers communautai res et sesmembres se sont transformés en marmitons d'un soir! La B ouffe-on-rit, c'est l'art d'apprêter des bons repas pas chers. Q uoi de plus agréable que de mettre la table et de partager un bon petit plat? Pour plusieurs personnes, dépourvues de véritables liens familiaux, c'est l'occasion de faire un brin de jasette, de faire la popote et de s'amuser. Q ue ce soit pour le party de $\mathrm{N}$ oël, ou celui de l'H alloween en passant par le pique-nique annuel au parc Strathcona, l'Alliance invite ses membres à choisir les activités pour l'année à venir. $N$ ous allons «aux vues», nous participons à des ateliers d'écriture, bref, nous sortons de notre coquille.

Au nom de l'amour et du réalisme, on nous destine souvent à une existence de reclus et de marginaux. La surprotection conduit inévitablement à une exclusion de nos responsabilités. Ballotés d'hôpitaux psychiatriques en foyers d'accueil, un grand nombre d'entre nous se trouvent engagés dans une voie sans issue. A fin de rendre la famille, les parents ainsi que les amis et amies plus conscients de toute la dynamique sociale, l'A lliance a aussi offert quelques conférences, entre autres sur la relation d'aide, la 
«L a connaissance donne le pouvoir. $\mathrm{E} n$ connaissant mieux nos droits, peut-être aurons-nous plus de pouvoir sur notre destinée.» défense des droits, les médicaments, les stratégies de survie et les coopératives d'emploi.

À l'Alliance, une chose est claire: nous sommes toutes et tous intéressés à faire quelque chose pour nous en sortir. Puisque la liberté passe par l'action, c'est à nous de poser des gestes qui nous permettront de nous réapproprier notre véritable pouvoir. La route est longue, souvent pénible. Affronter notre vie en toute connaissance de cause: voilà qui s'avère difficile pour la majorité d'entre nous qui oscillons entre le mieux et le pire. Q ue nous réserve l'avenir? D ifficile à dire quand nousfai sons le yo-yo entre le monde des chômeurs diplômés et celui des ateliers protégés. Le marché du travail est loin de répondre à nos besoins. La société a subi de profondes transformations. Elle brise ceux qui n'ont pas su s'y adapter. Plusieurs y laissent leur estime de soi. C omment remanier son emploi du temps quand on a perdu son emploi? L'Alliance se penche présentement sur la question. U ne des options envisagées est de mettre sur pied une coopérative de travail où règnerait une culture d'acceptation, de compréhension et de tolérance face aux problèmes de santé mentale.

Autre enjeu extrêmement important pour tous les membres de l'Alliance: la défense et le respect de ses droits. Q uels sont nos droits et nos recours une fois que nous nous ramassons en psychiatrie? Comme la protection en matière de santé mentale est synonyme d'évaluation psychiatrique et parfois de cure fermée, personne n'entend faire les frais d'une loi floue et complexe. U ne fois que les portes de I'hôpital se referment sur nous, une fois que les psychiatres brandissent l'alibi de la fameuse règle du secret professionnel, nous sommes en droit de nous demander si dans un tel climat, nous avons encore un mot à dire. $C$ 'est pourquoi l'Alliance travaille actuellement à la création d'un guide des droits pour les personnes ayant des problèmes de santé mentale. Le but de ce guide est de vulgariser quatre lois (santé mentale, consentement au traitement, décision au nom d'autrui et intervention). La connaissance donne le pouvoir. En connaissant mieux nos droits, peut-être aurons-nous plus de pouvoir sur notre destinée.

Évidemment, se lever pour éveiller la population est chose difficile mais non impossible. N ous avons tous des talents, des 
aptitudes et énormément de potentiel. N ous avons non seulement le droit maisle devoir de revendiquer notre place au sein de la communauté. À l'Alliance, nous sommes convaincus d'une chose: Iorsqu'une personne a vécu intensément une période difficile, elle comprend mieux ce que l'autre traverse. C ette complicité, cette connaissance vécue d'un problème sont la force de I'Alliance. Chez nous, personne ne se sent obligé d'être un bon intervenant, une bonne intervenante. $N$ ous pensons tout bonnement qu'il y a des interventions fort simples qui font beaucoup de bien. $\mathrm{N}$ ous nous rencontrons, parlons de ce que nous avons vécu, de ce que nous vivons. C ette solidarité, ce partage sont absolument nécessaires. Et qui d'autre mieux que nous peut enrayer les préjugés, l'indifférence, voire même l'intolérance.

C'est dans cette optique que l'Alliance a lancé E nsemble, son bulletin de liaison pour les francophones d' $\mathrm{O}$ ttawa- $\mathrm{C}$ arleton qui ont un vécu psychiatrique. Tribune d'expression de leurs différences en même temps que de leur créativité, cette publication leur permet également de sensibiliser la communauté: les personnes de l'Alliance ont un message à transmettre sur notre société, sur la difficulté d'y vivre et de s'y épanouir. D ans un monde où il faut arriver à tout prix, on passe trop souvent à côté de l'être humain. II n'est pas nécessaire pour les personnes ayant connu des périodes de détresse psychologique d'être condamnées à des conditions de vie à ce point décourageantes qu'elles aggravent leur état, leur maladie. L'Alliance permet d'échanger, de réfléchir et peut-être de trouver des pistes de solution par rapport à la souffrance. N ous avons des choses à dire: cet objectif est au coeur même de notre action.

S'informer pour mieux comprendre, c'est peut-être la règle

«L'histoire tend à prouver que les élans positifs viennent de la base; nos gouvernements sont habituelle ment plus habiles à réagir qu'à agir.» de base de toute lutte contre les injustices sociales. L'entraide commence souvent dans sa cour arrière. A mener les gens à tenir compte de l'existence de l' autre, c'est encore la meilleure mesure préventive contre les négligences de toutes sortes. II faut oser parler pour tous ceux et celles qui sont prisonniers d'euxmêmes autant que des camisoles de force chimiques. L'histoire tend à prouver que les élans positifs viennent de la base; nos gouvernements sont habituellement plus habiles à réagir qu'à agir. Prendre 
conscience de sa capacité de gérer son existence, de se prendre en main, voilà des habiletés essentielles par les temps qui courent. À I'heure des compressions dans le domaine de la santé, il est vital de créer des services de rechange, de préparer la communauté à assurer un support socioéconomique plus humain aux personnes vivant ou ayant vécu un épisode psychiatrique. À I'Alliance, nous apprenons à passer à travers au jour le jour. Et s'il nous arrive de nous sentir différent des autres, c'est tant mieux. $\mathrm{N}$ ous demeuronstousuniques malgré les efforts d'uniformisation de la société. 